\title{
Carnets
}

Revue électronique d'études françaises de l'APEF

Deuxième série - 13 | 2018

Corps, rythmes et voix : en/jeux littéraires et artistiques

\section{Césaire à l'écoute de la voix Majolè}

\section{Véronique Corinus}

\section{(2) OpenEdition}

Journals

Édition électronique

URL : http://journals.openedition.org/carnets/2556

DOI : $10.4000 /$ carnets. 2556

ISSN : 1646-7698

Éditeur

APEF

Référence électronique

Véronique Corinus, «Césaire à l'écoute de la voix Majolè », Carnets [En ligne], Deuxième série -

13 | 2018, mis en ligne le 31 mai 2018, consulté le 02 mai 2019. URL : http://journals.openedition.org/ carnets/2556; DOI : 10.4000/carnets.2556

Ce document a été généré automatiquement le 2 mai 2019.

\section{(c) (i) \&)}

Carnets est mis à disposition selon les termes de la licence Creative Commons - Atribution - Pas d'utilisation commerciale 4.0 International. 


\title{
Césaire à l'écoute de la voix Majolè
}

\author{
Véronique Corinus
}

Grand rocher éboulé infléchi du dedans

par l'invincible musique retenue prisonnière

d'une mélodie quand même à sauver du désastre

(Aimé Césaire, « Nous savoir », Ferrements, 1960)

1 Face au procès en créolophobie qui lui fut parfois intenté, Césaire usa d'une belle métaphore agronomique pour affirmer l'attachement à sa terre et à sa culture antillaises : «[P]rovignement, oui, dessouchement, non » (Césaire, 1987). Il revendiquait ainsi l'enracinement de son œuvre dans un territoire déterminé, la Martinique, inscription première qui favorise l'épanouissement vers d'autres espaces. C'est en somme un égal droit à l'île et au monde qu'il s'arrogea, refusant de sacrifier le particulier à l'universel, l'un étant le terreau de l'autre. Cette attention au local procède d'une "poétique du marcottage ${ }^{1}$ » dans laquelle la littérature orale antillaise tient un rôle important, bien qu'encore insuffisamment exploré.

La critique l'a longtemps minoré, ignorant souvent l'influence oralitaire dans la poétique césairienne, voire la contestant. Les travaux fondateurs de Lilyan Kesteloot et Lilian Pestre de Almeida ne sont pas encore totalement parvenus à battre en brèche les thèses bien installées d'Émile Yoyo (Yoyo, 1971) et de Raphaël Confiant (Confiant, 1993), qui ont largement contribué à construire l'image d'un Césaire acculturé, peu soucieux du patrimoine créole. Qu'on argue de sa méconnaissance supposée des traditions orales antillaises ou de son mépris pour un art qu'il jugerait fruste et désuet, c'est toujours l'image du lettré coupé de ses racines populaires qui s'impose. Césaire apparait ainsi comme celui qui est resté sourd à la voix du conteur qu'on appelle, en créole, le Majolè.

Celui dont le nom est étymologiquement rattaché à la parole s'apparente à une figure hyperbolique de la Voix. Le terme viendrait d'un mot normand désignant les caroncules de certaines volailles, notamment celles du coq. Par dérivation, il aurait fini par désigner la gorge des êtres humains. Une autre étymologie, fréquemment avancée, le rattache plus fortement encore à la voix, non plus de façon métonymique mais métaphorique : majolè serait issu de la racine «majô » (major i.e le maître, le chef) à laquelle serait adjoint le suffixe lè (air). Le mot signifierait littéralement : le maître de l'air i.e. du souffle, de la voix, 
de la parole. Quelle que soit l'origine retenue, son appellation fait du majolè un maitre de la parole par opposition au bajolè (le bavard, le hâbleur), qui parle pour ne rien dire ou pour dire des fadaises. La défiance que Césaire manifesterait à l'encontre du Majolè s'apparenterait ainsi à un refus de la voix même, de la source créole de la parole.

On ne saurait souscrire à une telle approche qui va à l'encontre de la réalité biographique et esthétique de Césaire. Durant son enfance passée sur les plantations au contact du peuple des cannaies, il a été immergé dans les traditions populaires et l'art du conte. Il en est si fortement marqué que, dès les années 1940, il procède à une entreprise de promotion de l'oralité antillaise dans son «Introduction au folklore antillais » (Césaire et Ménil, 1942). À la suite de La Revue du Monde Noir et de Légitime Défense, qui ont voulu revaloriser le patrimoine oral, il donne ses lettres de noblesse aux contes auxquels il ouvre les pages de la revue Tropiques. S'il les glose selon des orientations socio-historiques et psychologiques, il en perçoit également tout le potentiel esthétique.

5 Dans le poème « De forlonge » (Corps perdu, 1950), il inscrit le poète créole dans la filiation du conteur. Reproduisant les conditions d'énonciation de la contée traditionnelle, le Je lyrique se fait auditeur attentif du paroleur, dont il finira par prendre en charge le récit :

il y a en face de moi un paysan extraordinaire

ce que chante ce paysan c'est une histoire

de coupeur de cannes

han le coupeur de cannes

saisit la dame à grands cheveux

en trois morceaux la coupe

ah le coupeur de cannes

la vierge point n'enterre

la jette en morceaux

les jette derrière

ah le coupeur de cannes

chante le paysan et vers un soir de coutelas s'avance

sans colère

les cheveux décoiffés de la dame aux grands cheveux

font des ruisseaux de lumière

ainsi chante le paysan ("De forlonge », Corps perdu : 226-227)

En mettant en scène une veillée traditionnelle où un poète est à l'écoute du conteur, Césaire s'adonne à une parodie du poème de Daniel Thaly, "Conteurs martiniquais ", dont le Je lyrique rejoint également la ronde d'« un vieux noir (...), grave, hachant ses phrases ». Ce dernier délaisse le patrimoine créole pour lui préférer le répertoire de France, disant :

(...)

De « compère Lapin » l'histoire aux mille phrases

Et pourquoi « La plus Belle » était sous un tonneau.

Après Alibaba, c'est la Belle et la Bête.

Une femme des bois hochant sa lourde tête,

Dans son charmant récit évoquera Perrault.

Aux « Isles », on connaît Barbe-Bleue et Peau d'Âne,

Et j'aime, sur les flancs du vieux morne Pitaut,

Cendrillon raconté par un coupeur de cannes (Thaly, 1928 : 93)

7 Le conteur de « De Forlonge » s'écarte pour sa part de la tradition européenne. C'est cette mise à distance littéraire que traduit probablement le titre, expression archaïque 
signifiant « s'éloigner, laisser en arrière » et désignant singulièrement «le gibier traqué qui prend une grande avance sur les chiens » (Hénane, 2004: 61). Pour filer la métaphore, on pourrait dire, suivant la thèse de Lilian Pestre de Almeida, que le poème de Césaire tient à distance ce conte populaire français dans lequel une jeune vierge assassinée et enterrée revient réclamer vengeance (Pestre de Almeida, $2010: 209-222)$. Le lecteur peine à reconnaître ce conte d'autant plus qu'il est fortement modifié par Césaire. Le corps à corps meurtrier devient celui de l'homme des champs et «du végétal maudit des Caraïbes, la canne » (Ibid.: 211), cette dame à grands cheveux qu'on morcèle pour en répandre le sang poisseux. Les conséquences de ce «soir de coutelas » restent dans un implicite menaçant : la mise à mort initiale entraînera le châtiment de l'assassin, voué au joug éternel, comme si le geste du coupeur, dans ce conte devenu tragiquement étiologique, portait en lui sa propre damnation. Le conte occidental est réécrit pour s'ancrer résolument dans l'Histoire antillaise et raconter de façon «éloquente et pathétique, la grande misère de nos pères esclaves » (Césaire et Ménil, op.cit. : 7-11).

Pour en rendre compte, le poète adopte les éléments de l'art oratoire du majolè. Itération syntaxique et lexicale, parataxe, onomatopées et interjections : c'est une poétique de la Voix qui écrit ses premières lignes. Le Verbe du poète cherche à reproduire la voix du conteur, brisée par l'ahanement de l'effort et de la souffrance. Les enjambements, les parallélismes, le refrain - qui pourrait tout aussi bien être la ponctuation verbale des répondeurs qui interagissent avec le conteur - rendent compte du souffle et du rythme qu'il impose à sa diction. La métaphore qui assimile la canne à une femme en cheveux relève quant à elle d'une rhétorique propre aux tim-tim, ces devinettes traditionnelles qui saisissent le réel de façon imagée. Le trope relève d'un détour qui est le propre de l'esthétique de l'oralité selon Glissant qui signale que, dans la société plantationnaire où la parole est contrainte, le conte développe une parole de camouflage, "parole différée, ou déguisée, sous laquelle l'homme et la femme bâillonnés serrent ce qu'ils disent.» (Glissant, 1990 : 87).

9 L'art poétique de Césaire ne consiste pas uniquement à s'écarter de la tradition européenne par la ré-énonciation de son patrimoine mais également à se réapproprier le répertoire oral antillais. Sa poésie entre en relation intertextuelle de façon privilégiée avec des contes martiniquais collectés, transcrits et publiés par Lafcadio Hearn dans le recueil Trois fois bel conte (Hearn, 1932). Ce faisant, Césaire a recours à un intertexte hybride, la transcription, qui est une modalité de l'entre-deux, à la lisière de l'oral et de l'écrit. Elle occupe un statut intermédiaire dans le champ de l'intertextualité qui n'englobe pas une seule catégorie d'énoncés, l'intertexte pouvant être oral ou écrit, littéraire ou non littéraire. L'intertexte oralitaire sur lequel se fonde Césaire n'émane pas de l'oralité première mais seconde (Ong, 1983), voire tierce, privilégiant non l'immédiateté des discours produits en situation de performance mais le caractère différé des textes écrits.

10 L'hypotexte originel de Césaire est pourtant bien la parole vive que Hearn a patiemment collectée pendant deux ans. Déjà intéressé par les proverbes des Créoles louisianais qu'il a recensés dans Gombo Zhèbes (Hearn, 1885), Hearn se tourne lors de son séjour aux Antilles (1887-1889) vers un autre genre oral, les contes. Persuadé que jamais « aucun créole ne cultivera » (Hearn, cité par Denis, [1932] 1978 : 16) ce champ pourtant exceptionnel, il va cueillir à la bouche des gens du peuple ${ }^{2}$ des pièces de l'oralité créole. Il sollicite Abou, la fille de sa logeuse, qui « raconte des histoires créoles et des tims-tims» (Hearn, cité id.), Cyrillia, sa bonne, qui « contait à son corps les légendes dont frémissait sa pauvre vieille 
âme » (Hearn, cité ibid. : 17); il interroge également les gens croisés au hasard des ruelles escarpées de Saint-Pierre: Mme Robert la marchande de bout (cigares), prolixe en « légendes du temps jadis et (...) étranges histoires» (Hearn, cité id.), les enfants qui "[p]resque tous les soirs, un peu avant l'heure du coucher (...) se racontaient des histoires » (Hearn, $1926: 130$ ).

11 Face à cette masse orale, Hearn est confronté à l'épineuse question de la saisie de la parole vive à laquelle les folkloristes du XIX siècle ont fréquemment été soumis. Engoncé dans le profond malentendu (Belmont, 2007 : 103-125) partagé par les premiers collecteurs, il cherche dans l'oralité les critères de l'écrit (unicité, fixité et réitérabilité du Verbe). Aussi est-il gêné par le foisonnement et la "mouvance incessante » (Id.) d'une parole qui jaillit librement et que la dictée contraint. Il s'en plaint avec une amertume pleine de condescendance :

J'ai réussi à m'en faire dicter plusieurs, afin de pouvoir ensuite les écrire (...) Afin de garder toute leur simplicité primitive et la naïveté humoristique des détails, il faudrait les noter sténographiquement à mesure qu'on vous les raconte; elles perdent beaucoup par le procédé plus long de la dictée. L'esprit simpliste du conteur est embarrassé par les interruptions et contraintes inévitables qui résultent de la dictée; le conteur perd sa verve, se lasse et raccourcit volontairement la narration afin de terminer cette tâche aussi vite que possible. Il lui semble pénible de répéter une phrase plus d'une fois, du moins de la même façon, tandis que des questions fréquentes irritent parfois le plus docile à un point inouï. (Hearn, $1926: 130$ )

12 Afin de pourvoir aux supposées «défaillances du conteur» (Denis, op.cit.: 19), il intervient sur les histoires qu'il a collectées avec beaucoup de minutie mais qu'il a, de l'avis de son traducteur Serge Denis, « remaniées, composées, écrites » (Ibid. : 24). L'étude de son manuscrit montre que toute digression voire confusion a été éliminée pour canaliser le récit dans une linéarité de bon aloi. Des points d'interrogation notés au crayon signalent « les passages qui lui paraissent superflus et qu'il hésite à conserver " (Id .). C'est là le témoignage évident de sa volonté de ré-agencer la matière orale, quitte à la tronquer. Tandis que sont gommées les hésitations et scories verbales qui émaillent toute performance, sont ajoutés des éléments qui rendent le récit plus lisible. Loin d'être « un simple copiste » (Id.) de l'oralité, Hearn se révèle être un auteur qui substitue sa plume d'écrivain à la voix du conteur dont le caractère foisonnant et débordant est désormais maîtrisé.

13 C'est à cette transcription qui assourdit la Voix du conteur que Césaire se réfère. La médiatisation graphique s'est cependant avérée essentielle puisqu'elle a su le sensibiliser à la poéticité de l'oralité créole. Reconnaissant cette initiation fondamentale, Césaire rend un hommage appuyé ${ }^{3}$ au journaliste helleno-irlandais dans deux poèmes de Ferrements (1960): «Statue à Lafcadio Hearn» et "Beau sang giclé». Disposés en diptyque, ils s'apparentent à un double tombeau élevé à Hearn dont les collectes de contes populaires ont su restituer à sa mémoire le noir verbe. Le recueil du folkloriste étranger, bien qu'altérant la voix du conteur, lui fournit les hypotextes oralitaires qui vont nourrir sa poétique.

14 La production de Césaire tisse des liens intertextuels privilégiés avec quatre contes collectés par Lafcadio Hearn: "Conte Colibri», «Soucouyan», "Yé» et «Nanie Rozette ». Ce dernier récit, qui clôture Trois fois bel conte, est explicitement évoqué dans le poème «Statue de Lafcadio Hearn » : 
Nanie-Rosette mangeait sur un rocher

le diable volait autour

oint de graisse de serpent

d'huile de trépassés (« Statue à Lafcadio Hearn », Ferrements : 334)

Les quatre vers reprennent de façon elliptique le conte d'avertissement connu aux Antilles sous le titre générique de « La Roche du Diable». Il raconte comment fut châtiée la gourmandise d'une fillette. Cette dernière, pour ne pas avoir à partager son repas, s'éloigne de sa maison et s'installe, afin de le déguster, sur un rocher fiché au milieu d'une rivière. Mais hélas elle reste prisonnière de la pierre, y étant comme " collée à la colle forte » (Hearn, [1932] 1978: 97). La roche s'avère être un lieu maléfique où le Diable rassemble sa troupe infernale :

C'est là que se faisait le Sabbat: la ronde de Satan; là que s'assemblaient Zombis, Soucouyan, Loup-Garou, Agoulou.

Les Engagés de Satan n'étaient guère contents, n'est-ce pas ?

Le Rocher, c'était la table où le Diable les enduisait de graisse de serpent, d'huile de trépassés, de phosphore, et des mille ingrédients qui, la nuit, donnent à Zombi sa clarté. (Hearn, [1932] 1978 : 98).

Césaire reprend dans les vers de "Statue de Lafcadio Hearn» les deux onguents sataniques, dont la singularité retient son attention. Il fait de la " graisse de serpent ${ }^{4}$ » et de l'« huile de trépassés ${ }^{5}$ » les métonymies des débordements et dérives du Sabbat qui menacent Nanie. La maison que sa mère s'empresse de faire construire autour de la fillette pour la protéger n'y changera rien : le Diable réussit à la tromper en imitant la voix maternelle et « la dévora comme une chevrette » (Hearn, [1932] 1978 : 102).

Dans son « Introduction au folklore martiniquais », Césaire rattache l'histoire de Nanie au " cycle de la faim» qui regroupe les contes créoles traitant d'une même réalité sociohistorique : la famine et la malnutrition auxquelles les esclaves étaient continuellement confrontés. Leurs contes expriment dès lors l'angoisse de la disette par l'image inversée de la gourmandise, l' «obsession des ventres vides", étant toujours à l'origine du «fantasme de bombance » (Césaire et Ménil, op. cit. : 7). Les rêves de ripailles Nanie sont en somme l'expression détournée de la frustration impuissante d'une enfant affamée, aux prises avec la violence d'un système oppressif, fondé sur un principe de privation généralisé. L'esclavagisme ôte les moyens de subsistance matériels nécessaires à la préservation physique tout comme il dépossède de celui essentiel à la préservation psychique : la liberté.

18 La gourmandise n'est plus dès lors à envisager uniquement selon le prisme catholique qui en fait un péché capital coupant l'individu des hommes et de Dieu par sa transgression des lois sociales et divines. Dans le cadre plantationnaire, elle traduit une propension de l'esclave à se dresser contre les règles coercitives imposées par le maître. Concupiscible d'un nouveau genre, elle témoigne d'un désir de possession autrement plus subversif : la liberté. La voracité de Nanie apparaît non seulement comme une volonté de se soustraire à la pénurie alimentaire, mais aussi à l'enfermement plantationnaire. Pour déguster son repas, elle n'hésite d'ailleurs pas à franchir l'enceinte de sa maison et à s'enfoncer très profondément dans les bois :

Manzelle remplit son assiette de toutes sortes de bonnes choses : migan, avocats et farine-manioc, court-bouillon-crabe cirique, poingole, morue et chatron (sic) en pimentade ; et s'en alla hors de la maison chercher la solitude (...) Enfin, enfin, elle trouva loin, loin, loin, au creux d'un ruisseau, un rocher grand comme une maison. Elle eut assez de courage pour monter tout dessus, en haut. 
Assise là, bien comme il faut, elle mangea son content ; personne n'allait venir, si

haut, lui demander ses miettes. (Hearn, [1932] 1978 : 96)

19 L'escapade dans les bois de Nanie s'apparente à une échappée marronne. La fillette s'élance dans les bois pour fuir la servitude, faisant de son péché de gourmandise l'euphémisation d'une convoitise plus transgressive encore.

Mais celle qui courait les bois est immobilisée par la roche qui la retient prisonnière, et la remet symboliquement à la chaîne en la collant à ses parois. En cela, Nanie subit le sort des oiseaux destinés à être mis en cage, que l'on attrapait au moyen d'une glue, faite de la sève visqueuse de l'arbre à pain. La maison que sa mère fait construire autour d'elle ne fait que renforcer sa claustration, en l'empêchant définitivement de déambuler. Son désir d'émancipation reçoit un châtiment plus extrême encore : la mort qui vient sanctionner, comme le stipule le Code Noir, le marronnage répété. Nanie est punie par là où elle a péché : celle qui rêvait de dévorer le monde est dévorée. L'exécuteur des basses œuvres est d'ailleurs le Diable, figure traditionnelle du colon dans les contes créoles.

Par son dénouement funeste, le conte populaire diffuse une vision pessimiste, affirmant la destinée fatale du peuple noir, condamné à jamais à la soumission. Conte d'avertissement mettant en garde contre toutes velléités émancipatrices, il distille une morale conservatrice qui dit la vanité des luttes inévitablement sanctionnées par la défaite. Il exhorte chacun à accepter l'organisation sociale et raciale que l'Histoire a imposé dans les colonies d'Amérique.

Ne pouvant souscrire au discours de résignation du conte populaire, Césaire le métamorphose. Afin de le soustraire au fatalisme, il s'adonne à sa réécriture poétique par la troncation de sa situation finale : dans "Statue de Lafcadio Hearn », la fillette n'est pas vaincue par son geôlier. Celle qui était collée à son rocher se redresse, emportée par le mouvement ascensionnel décrit dans le vers précédent : "Yé grimpa au palmier ». Nanie est ainsi associée à l'élan d'un second personnage du recueil Trois fois bel : Yé, le «Bitaco typique» (Hearn, 2004: 408) des contes populaires. Césaire voit dans ce "nègre montagnard [ou] Noir campagnard dont les Noirs citadins aiment à se moquer » (Id.) le double masculin de Nanie. Comme elle, il est victime de sa gourmandise. Pour le punir de sa voracité, le Diable élit domicile chez lui et dévore chaque jour la nourriture de sa famille; non content de l'affamer, il l'oblige à manger ses excréments. L'incompétence de Yé l'empêche de se débarrasser de l'intrus si bien que sa famille ne doit son salut qu'à l'ingéniosité de son cadet.

Césaire ne se réfère pas à cette figure dérisoire et pathétique de Yé, symbole de l'homme noir « écrasé par une société inique » (Césaire et Ménil, op.cit. : 8) dont Trois fois bel conte fait le portrait déplorable. Le poème entre en relation intertextuelle avec un commentaire de Hearn qui réévalue le personnage traditionnel :

Pauvre Yé, tu vis encore pour moi d'une façon intense ailleurs que dans ces histoires de boire et de manger qui révèlent si cruellement la longue faim de ta race d'esclaves. Car je t'ai vu couper la canne à sucre sur les flancs de montagnes bien au-dessus des nuages. Je t'ai vu grimper de plantation en plantation, le coutelas à la main; je t'ai vu grimper à travers les bois grouillants de serpents jusqu'à quelque cratère éteint pour cueillir un choux palmiste, toujours affamé, toujours sans ressources. (Hearn, 1926 : 138)

Le vers elliptique du poème condense l'énoncé métatextuel qui transforme Yé, le pauvre bougre, en un homme courageux et déterminé. Poursuivant son héroïsation, le poème en 
fait un insurgé. Son coutelas annonce les « longs rasoirs » des esclaves du 22 mai 1848 qui mirent la ville de Saint-Pierre à feu et à sang et précipitèrent l'abolition ${ }^{6}$ :

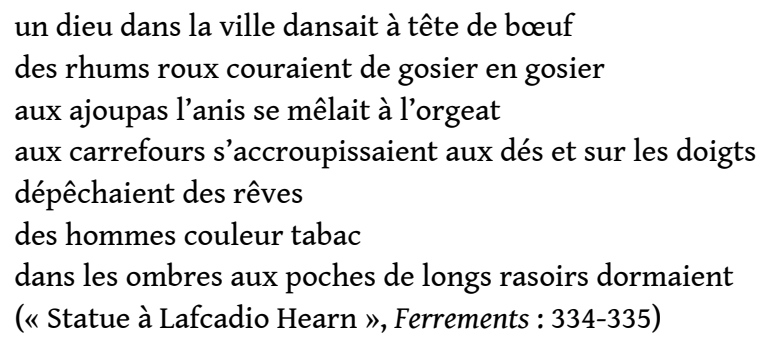

En incluant le personnage de Nanie dans un tel contexte révolutionnaire, Césaire transfigure la figure de la Marronne vaincue en Insurgée farouche. Elle devient une virago , empreinte de la vitalité de ceux qui prirent les armes pour défendre leur liberté. L'héroïsation de la Maronne du conte montre toute la distance que Césaire sait prendre avec la parole du conteur, porteuse d'un inconscient collectif aliéné qu'il s'agit de transformer. Son travail de réécriture n'est pas une simple opération d'assimilation patrimoniale ni d'hommage inconditionnel. Prendre langue avec le conteur s'apparente à un véritable détournement de sa voix afin d'appeler au redressement de toute une communauté. La réécriture du conte est une refondation pour Césaire qui s'enorgueillit d'avoir « forgé [s]a mythologie » (Césaire, 1994).

Le conte patrimonial réénoncé et resémantisé par Césaire offre au poète de nouvelles potentialités esthétiques. Tour à tour Marronne insurgée et Marronne châtiée, le personnage de Nanie impose les motifs de sa geste : la femme en mouvement et la femme figée, l'eau, le fer coercitif et trompeur, la prison de pierre, etc. Le conte poétisé fonctionne comme un intratexte, dans lequel le poète puise le matériau indispensable à la fabrique de métaphores oralitaires percutantes empruntant leurs éléments constitutifs au patrimoine oral antillais réinventé. Leur hermétisme peut être décrypté dès lors que l'on possède l'arrière fond culturel qui préside à leur composition.

Le conte patrimonial permet ainsi d'expliquer certaines incongruités du poème des Armes miraculeuses, « La Forêt vierge », qu'une silhouette énigmatique traverse :

Où allez-vous ma femme marron ma restituée ma cimarronne il vit à pierre fendre et la limaille et la grenaille tremblent leur don de sabotage dans les eaux et les saisons Où allez-vous ma femme marron ma restituée ma cimarronne le cœur rouge des pierres les plus sombres s'arrête de battre quand passent les cavaliers du sperme et du tonnerre

(« La forêt vierge », Les armes miraculeuses : 110)

La femme marronne surgit brutalement et avance dans le poème, telle une «ombre libérée d'un joug, en rupture de servitude, rétablie dans ses droits » (Hénane, $2008: 230$ ). Elle déambule dans un paysage saccagé. Le texte désarticulé rend compte de sa confusion apocalyptique, déroutant le lecteur par ses incongruités syntaxiques, ses incohérences lexicales, sa structure labyrinthique. L'insurrection à laquelle la femme marronne a participé cause une dévastation qui a affecté l'espace et jusqu'au langage.

La Marronne semble bien être un avatar de Nanie dont elle partage le destin. Sa mésaventure est rappelée par diffraction sémantique. Les mots "pierre(s) » et «eau " permettent de reconstituer le cadre minéral dans lequel va être commis le meurtre. La mention de « la limaille et la grenaille » évoque l'action du forgeron qui affine la langue trompeuse du Diable désormais apte à opérer son «don de sabotage ». Le Diable sera encore lié au métal, par l'allusion à venir aux farouches "euphorbes fétiches", ces 
gardiens végétaux à la solde de leur loa tutélaire, Oggùn, dieu forgeron vaudou manieur de feu et de fer. Aussi redoutable que les quatre chevaliers de l'Apocalypse, le Diable est, dans le présent extrait, identifié aux « cavaliers du sperme et du tonnerre ", la dévoration étant une euphémisation du viol. Viol tout à la fois d'une femme, d'un peuple, d'une culture. La Marronne échappe cependant à sa cruauté, apparaissant au terme du poème dans un paysage renaissant :

(...) ne manque pas de chanter la forêt vierge jaillie du silence de la terre de mes yeux à moi-même aléoutiens et c'est ainsi le saute-mouton salé des pensées hermaphrodites des appels de jaguars de source d'antilope de savanes cueillis aux branches de mes yeux pour toi aussi aléoutiens lancés à travers leur première grande aventure : la cyathée merveilleuse sous laquelle s'effeuille une jolie nymphe parmi le lait des mancenilliers et les accolades des sangsues fraternelles.

( La forêt vierge », Les armes miraculeuses : 111)

Échappant à la roche diabolique, la Marronne devenue nymphe s'est redressée, gagnée par la verticalité d'un paysage arboré. La double allusion aux îles Aléoutiennes, archipel volcanique au Sud-Ouest de l'Alaska, substitue à la claustration de la pierre collante la libération de la roche éruptive.

31 Le symbolisme de la roche, emprunté au conte poétisé, gardera chez Césaire sa dualité. Elle est tout à la fois ce qui contraint et ce dont on s'affranchit, fidèle en cela à Nanie qui s'est arrachée de la roche qui la retenait. Si elle est geôle à l'instar de ces pierres qui deviennent " prison d'escargots » ("Conspiration », Moi...laminaire : 435) ou du rocher qui retient Prométhée cloué à ses parois («Dérisoire », Comme un malentendu de salut: 510), elle est aussi espace de liberté. Elle prend alors l'aspect d'une pierre brisée, «pierre qui s'émiette en mottes» ("En vérité», Ferrements: 379), voire pierre taillée dont le tranchant dit la pugnacité du rebelle :

(...) je t'énonce

FANON tu rayes le fer

tu rayes le barreau des prisons tu rayes le regard des bourreaux guerrier-

silex

vomi par la gueule du serpent de la mangrove

(« Par tous les mots-silex », Moi... laminaire : 394.395)

Ainsi en va-t-il dans « le Rocher de la femme endormie ou belle comme l'exaspération de la sécession " ("Configurations », Comme un malentendu de salut : 519-522). Le titre renvoie au Rocher de la femme couchée, lieu-dit situé sur le Morne Larcher, au sud-ouest de la Martinique, où Césaire aimait à venir s'entretenir, par-delà la mort, avec sa défunte femme, Suzanne. Sans doute son épouse figée dans la mort lui semblait-elle être devenue cette femme de pierre immobile que dessine le relief de l'ancien volcan éteint :

Rescapée rescapée

C'est toi la retombée

D'un festin de volcans

D'un tourbillon de lucioles

D'une fusée de fleurs d'une fureur de rêves

(« Le Rocher de la femme

endormie ou belle comme l'exaspération de la sécession », Comme un malentendu de salut : 514)

La femme couchée, prise dans une torpeur minérale et baignée par les eaux de la mer Caraïbe, est semblable à une Nanie pétrifiée, immobilisée par la roche qui la soumet. Comme elle, la Femme endormie voit ses velléités d'affranchissement entravées par la présence d'un gardien terrifiant : 
Plus d'une fois j'ai enhardi la vague

A franchir la limite qui nous sépare toujours

Mais le dragon gouverne le cap de cette eau interdite

Même si c'est souvent en inoffensif caret-plongeur

Qu'il survient respirer à la surface maudite.

(«Le Rocher de la femme endormie ou belle comme l'exaspération de la sécession »,

Comme un malentendu de salut : $514-515$ )

En cela, elle est une figuration tragique de la léthargique Martinique qui doit trouver les voies de sa liberté :

Très pure loin de toute cette jungle

La traîne de tes cheveux ravivée

Jusqu'au fond de la barque solaire

Exaspération de la sécession.

(«Le Rocher de la femme endormie ou belle comme l'exaspération de la sécession »,

Comme un malentendu de salut : 514)

Le désir d'émancipation est traduit par le mouvement d'arrachement que le préfixe du verbe exaspérer exprime, associant la liberté à la nécessité de s'extirper d'un lieu où l'on est retenu par force.

Le figement présent n'est pas résignation : il dit tout à la fois l'éruption passée qui le fit naître et le jaillissement futur qu'il couve. Toute pierre, en dépit de son immobilité, porte en elle l'esprit de sédition pour Césaire qui rappelle que « toute la Martinique est née de volcan, du volcanisme sous-marin (...) c'est une terre qui est née des convulsions; une terre crachée des entrailles les plus profondes de la terre » (Césaire,2003:27). Et d'ajouter que ce « côté flégréen a beaucoup influencé [s]on imaginaire » (Id).

Le conte, parce qu'il évoque avec la « surrection d'un rocher » (« Configurations », Comme un malentendu de salut, 1994: 522) le redressement d'un peuple, a la charge de dire l'insurrection à venir, libératrice. Il devient, par sa ré-énonciation, poésie, qui elle-même est surgissement d'une parole démiurgique : «Alors quid de la poésie? Il faut toujours y revenir : surgie du vide intérieur, comme un volcan qui émerge du chaos primitif, c'est notre lieu de force, la situation éminente d'où l'on somme ; magie ; magie ; magie » (Césaire, cité par Kesteloot et Kotchy, $1973: 6$ )

La parole du majolè, qu'on nomme également en créole le mantò (quimboiseur, sorcier), est propice à une telle magie, c'est sans doute la raison pour laquelle Césaire prêta une oreille attentive à sa voix. Une voix dont le timbre lui revient paradoxalement à travers les pages d'un recueil, dans les années 40 , à son retour au pays natal. La lecture de Lafcadio Hearn lui fit redécouvrir le conte oral depuis longtemps tapi dans sa mémoire, attendant la transmutation livresque pour se muer en matériau littéraire. La médiatisation du conte par l'écrit étranger participa à l'élaboration de sa poétique, le poussant à enrichir son écriture de métaphores oralitaires éruptives. Ainsi son individualité entra-t-elle davantage en résonnance avec sa communauté, «le vieux fond ancestral» (Césaire, 1945 : 167) faisant émerger le Moi. La voix singulière du Poète put alors prendre en charge plus résolument encore la voix collective de son peuple. Ce faisant, il ne fait pas allégeance à son paroleur mais prend langue avec lui afin de faire, ensemble, évoluer les consciences.

Peut-être doit-il à ce rapprochement avec le Majolè la disparition du bégaiement dont il était affecté et qui lui avait valu, de la part de ses élèves du lycée Schoelcher, le surnom moqueur de «lézard vert ». Le zézaiement cessa brusquement d'entraver son élocution à son retour d'Haïti en 1944, alors qu'il s'était immergé durant sept mois dans la culture 
haïtienne. Entendit-il là-bas la voix vive du majolè ? Il fréquenta du moins le cercle de Jean-Price Mars qui exhortait les écrivains indigénistes à revenir à l'oralité primordiale. Quoi qu'il en soit, le jeune Césaire rentra en Martinique métamorphosé. Doté, selon le jeune Joseph Zobel, d'« [u]ne voix, un ton jamais entendus » (Alliot, 2008 : 92). Il devient dès lors l'orateur que l'on sait, capable d'enflammer les foules et de faire trembler l'Assemblée Nationale. Il devient surtout l'auteur d'une œuvre tonitruante qui l'imposera dans la littérature mondiale.

\section{BIBLIOGRAPHIE}

ALLIOT, David (2008). Aimé Césaire, Le Nègre universel. Paris : Infolio.

BELMONT, Nicole (2007). « Elle ne peut lire une seule lettre. Approche d'un malentendu », Cahiers de littérature orale [En ligne], 62 | 2007, mis en ligne le 17 mars 2013, consulté le 02 septembre 2017. URL : http://journals.openedition.org/clo/1221; DOI : 10.4000/clo.1221

CÉSAIRE, Aimé et MÉNIL, René (1942). «Introduction au folklore antillais », Tropiques, $\mathrm{n}^{\circ} 4$, janvier 1942.

CÉSAIRE, Aimé (1945/1978). « Poésie et connaissance », Extraits d'une communication présentée au Congrès de Philosophie de Port-au-Prince (Haïti), parue dans la revue Tropiques, $\mathrm{n}^{\circ} 12$, janvier 1945, rééditée par Jean-Michel Place, 1978, pp. 157-170.

CÉSAIRE, Aimé (1962). « Réflexions sur la poésie. Lettre à Lylian Kesteloot », Lilyan Kesteloot, Aimé Césaire. Seghers, coll. « Poètes d'aujourd'hui ».

CÉSAIRE, Aimé (1987). « Conférence des peuples noirs de la diaspora », prononcée à Miami, le 26 février 1987.

CÉSAIRE, Aimé (1994). « Entretien avec Gilles Anquetil », Le Nouvel Observateur.

CÉSAIRE, Aimé (1994/2006). La poésie. Édition établie par Daniel Maximin et Gilles Carpentier, Paris : Édition du Seuil. Toutes les références des poèmes cités dans l'article renvoient à cette édition.

CÉSAIRE, Aimé (1970). « La forêt vierge », Les armes miraculeuses [Paris, 1946], pp. 109-111.

CÉSAIRE, Aimé (1950). « De forlonge », Corps perdu [Paris : Éditions Fragrance, 1950], pp. 226-227.

CÉSAIRE, Aimé (1991). « En vérité », Ferrements [Paris : Seuil, 1960], p. 379.

CÉSAIRE, Aimé (1991) « Statue à Lafcadio Hearn », Ferrements [Paris : Seuil, 1960], pp. 334-335.

CÉSAIRE, Aimé (1991). « Beau sang giclé », Ferrements [Paris : Seuil, 1960], pp. 336.

CÉSAIRE, Aimé (1982). « Par tous les mots-silex », Moi... laminaire. Paris : Seuil, 394.395.

CÉSAIRE, Aimé (1982). « Conspiration », Moi... laminaire. Paris : Seuil, 1982, pp. 435-436.

CÉSAIRE, Aimé (1994). « Dérisoire », Comme un malentendu de salut. Paris : Seuil, p. 510. 
CÉSAIRE, Aimé « Le Rocher de la femme endormie ou belle comme l'exaspération de la sécession », Comme un malentendu de salut [Paris : Seuil, 1994], p. 514-515.

CÉSAIRE, Aimé « Configurations », Comme un malentendu de salut [Paris : Seuil, 1994], 519-522.

CÉSAIRE, Aimé (2003). « Genèse d'une pensée », entretien avec Jacqueline Leiner (1996), in Jacqueline Leiner (dir.), Le terreau primordial (tome 2). Gunter Narr Verlag Tübingen.

CONFIANT, Raphaël (1993). Aimé Césaire, une traversée paradoxale du siècle. Paris : Stock.

GLISSANT, Édouard (1990). Poétique de la Relation. Paris : Gallimard.

HEARN, Lafcadio (1885). Gombo Zhèbes. Little Dictionary of Creole Proverbs, selected from six creole dialects, translated into French and English, with notes, index and remarks upon the creole idioms of Louisiana, by Lafcadio Hearn, New-York: édit. Will H. Coleman.

HEARN, Lafcadio (1926). Contes des Tropiques. Traduit de l'anglais par Marc Logé, Paris : Mercure de France, p. 130.

HEARN, Lafcadio (1929). Esquisses martiniquaises. Traduit de l'anglais par Marc Logé, Paris : Éditions Mercure de France, coll. « Auteurs étrangers ».

HEARN, Lafcadio (1890/1923). Youma, roman martiniquais. Paris : Mercure de France ; première édition : Harpers and Brothers.

HEARN, Lafcadio (1932/1978). Trois fois bel conte. Traduit de l'anglais par Serge Denis, Avec le texte original en créole antillais, Paris : Éditions Mercure de France, Collection d'auteurs étrangers ; Calivran Anstalt, B.P. 183, Vaduz, Liechtenstein.

HEARN, Lafcadio (2004). Aux vents caraibes. Hoëbeke, coll. "Etonnants Voyageurs".

HÉNANE, René (2004). Glossaire des termes rares dans l'œuvre d'Aimé Césaire. Paris : Jean-Michel Place. HÉNANE, René (2008). Les armes miraculeuses » d'Aimé Césaire. Une lecture critique. Paris :

L'Harmattan.

KeSTELOOT, Lilyan (1962). Aimé Césaire. Seghers, coll. « Poètes d'aujourd'hui ».

Kesteloot, Lilyan et Kotchy, Bernard (1973). Aimé Césaire, l'homme et l'œuvre, Présence africaine, coll. « Approche ».

LEMAIRE, P. L. et PAUQUET, H. L. E. (1864). Histoire naturelle des oiseaux exotiques. Paris: F. Savy.

ONG, Walter J. (1982). Orality and Literacy, the Technologizing of the World. London \& New York,:Methuen.

ZUMTHOR, Paul (1983). Introduction à la poésie orale. Paris : Seuil.

PESTRE DE ALMEIDA, Lilian (2010). « 'De forlonge' ou à partir de l'oralité traditionnelle », Samia Kassab-Charfi (dir.), Altérité et mutations dans la langue. Pour une stylistique des littératures francophones. Bruxelles : Academia Bruylant.

THALY, Daniel (1928). « Conteurs martiniquais », Chants de l'Atlantique, suivi de Sous le ciel des Antilles . Paris : Garnier.

YOYO, Emile (1971). Saint John-Perse et le conteur. Paris : Editions Bordas, coll. "Etudes". 


\section{NOTES}

1. On voit comme la pensée de Césaire entre en convergence avec la notion d'identité rhizomatique, développée par Deleuze et Guattari et reprise par Glissant, en usant de l'image du provignement ou marcottage, procédé de reproduction des végétaux, consistant à faire développer des racines à une tige pour obtenir, après séparation de la plante mère, un plant autonome.

2. Il faut préciser que quelques histoires furent notées à son intention par des Créoles.

3. La reconnaissance prit également la forme d'un hommage du maire qui fit donner à la place du Marché de l'asile situé boulevard du Général de Gaulle, le nom de Lafcadio Hearn. Nombre de chercheurs considèrent, à l'instar de Césaire, que Lafcadio Hearn a largement contribué à la revalorisation du patrimoine oral créole. C'est ainsi que le folkloriste fut gratifié, tout comme Ina Césaire et Gilbert Gratiant, du titre de « Mèt a manniòk de l'oralité martiniquaise » lors du Symposium International et pluridisciplinaire sur le rôle du conte et de l'oralité dans l'imaginaire, l'esthétique littéraire et artistique.

4. On se servait auparavant de la graisse de serpent pour éloigner les animaux dangereux. Cette pratique est toujours utilisée aux Antilles.

5. Sa bonne Cyrillia explique à Lafcadio Hearn que les «moun-mô ne sont pas des Zombis. Les Zombis vont partout ; les moun-mô demeurent dans le cimetière, excepté la nuit des morts : cette nuit-là, ils rendent visite à leurs parents, ils vont partout. » (Hearn, 1929 : 56).

6. Hearn raconte l'insurrection de 1848 dans Youma, roman martiniquais (Paris, Mercure de France, 1923 ; première édition : Harpers and Brothers, 1890) dont Césaire reprend des fragments dans son poème.

\section{RÉSUMÉS}

La critique a longtemps voulu ignorer le rôle joué par la littérature orale antillaise dans la poétique de Césaire, considérant qu'il se serait détourné avec mépris de l'art du conteur. Il apparaît, a contrario, qu'un véritable poétique de la voix est à l'œuvre dans sa création qui s'est inspirée de l'art du majolè (i.e. conteur antillais) pour innerver son écriture de son souffle, de sa langue et de ses motifs. La ré-énonciation poétique de la tradition orale passe paradoxalement par les transcriptions publiées dans le recueil de Lafcadio Hearn, Trois fois bel conte (1932). L'oralité seconde substitue alors ses signes graphiques à la labilité de l'oralité première, avant d'être transmuée en voix poétique. Voix poétique qui ne se fait pas mimétique de celle du conteur, dont Césaire se réapproprie le discours pour l'infléchir vers une ode à l'émancipation. L'exemple du conte «Nanie Rozette » permet de percevoir le travail de réécriture de l'oralité antillaise engagé par Césaire.

Césaire's poetic contribution to the Caribbean oral literature has been for longtime ignored by literary criticism. He was supposed to be contemptuous of the storyteller's art. On the contrary, it appears that a real poetic of the voice is the one which conveys the spirit of the text inspired by the art of the majolè (Caribbean storyteller). Césaire has enriched his work by depicting the storyteller's breath, language and tales. He transforms oral tales into poems, with unexpected 
reference to Lafcadio Hearn's transcriptions, published in a storybook: Trois fois bel conte (1932). His transcriptions substituted spoken words for written words which become poetry. The poet's voice does not imitate the storyteller's voice. Césaire steals his words to turn them into a song for liberty. With the tale entitled "Nanie Rozette", we can study Césaire's work on Caribbean orality.

INDEX

Mots-clés : Césaire (Aimé), Hearn (Lafcadio), contes créoles, réécriture, intertextualité

Keywords : Césaire (Aimé), Hearn (Lafcadio), Carribean tales, rewriting, intertextuality

\section{AUTEUR}

VÉRONIQUE CORINUS

Université Lumière Lyon 2 - Laboratoire Passages XX-XXI Institut International pour la francophonie

veronique.corinus[at]univ-lyon2.fr 